\title{
Efeito dos parâmetros de retificação no acabamento superficial de cerâmicas avançadas
}

\section{Effect of Grinding Parameters on Surface Finishing of Advanced Ceramics}

\author{
Ana Cláudia Rodrigues ${ }^{1}$, Renan Negri Franco de Souza ${ }^{1}$,Otavio Ferrarini Galisa ${ }^{1}$, \\ Thiago Valle França ${ }^{1}$, Eduardo Carlos Bianchi ${ }^{1}$, Cesar Renato Foschini ${ }^{1}$
}

\footnotetext{
${ }^{1}$ Universidade Estadual Paulista, Unesp, Faculdade de Engenharia de Bauru, Dept. de Eng. Mecânica, Av. Eng. Luiz Edmundo C. Coube 14-01, CEP 17033-360, Bauru, SP, Brasil.

e-mail: anaclaudiarodrigues22@yahoo.com.br; otavio10ferrarini@hotmail.com; renan.franco7@hotmail.com; thiagovf@feb.unesp.br; bianchi@feb.unesp.br; cfoschini@feb.unesp.br
}

\begin{abstract}
RESUMO
As cerâmicas avançadas apresentam uma ampla aplicação, desde componentes para a indústria eletrônica até implantes e próteses. Nas últimas décadas a biocerâmica vem chamando atenção em aplicações como próteses ósseas e implantes dentários, pois apresentacaracterísticas de material bioinerte, bioativo, com boa biocompatibilidade, além de alta resistência à compressão. Neste trabalho, estudou-se o efeito dos parâmetros de retificação em cerâmicas de alta alumina preparadas com três granulometrias diferentes, através da análise do acabamento superficial destas cerâmicas. Durante o processo de retificação, os parâmetros variados foram: velocidade da peça $(55 \mathrm{~mm} / \mathrm{s}$ e $250 \mathrm{~mm} / \mathrm{s})$ e profundidade de corte $(5 \mu \mathrm{m}$ e $60 \mu \mathrm{m})$. Além destes parâmetros, empregaram-se dois tipos de lubri-refrigeração: convencional e MQL (Mínima Quantidade de Lubrificante). Os resultados obtidos através de medidas de rugosidade da superfície retificadae análises estatísticas mostram que houve uma redução na rugosidade das peças com granulometria media e fina quando se utilizou a lubrirefrigeração MQL. As cerâmicas de granulometria fina retificadas com velocidade da peça de $55 \mathrm{~mm} / \mathrm{s}$ e profundidade de corte de $60 \mu \mathrm{m}$ apresentaram rugosidade inferior a 0,28 $\mu \mathrm{m}$ usando o sistema de lubrirefrigeração MQL. Porém quando retificadas em velocidades maiores utilizando MQL as cerâmicas de granulometria mais fina apresentaram um aumento nos valores de rugosidade.
\end{abstract}

Palavras-chave: cerâmica, alumina, retificação.

\begin{abstract}
The advanced ceramics shows a wide application, from components for the electronic industry to implants and prostheses. In the last decades the bioceramic has been drawing attention in applications such as bone prostheses and dental implants because it presents bioinert and bioactive material characteristics, with good biocompatibility, and high compressive strength. We studied the effect of grinding parameters for ceramics with high alumina, prepared with three different particle sizes by examining the surface finish of these ceramics. During the grinding process, the parameters were: table speed $(55 \mathrm{~mm} / \mathrm{s} 250 \mathrm{~mm} / \mathrm{s})$ and the depth of cut $(5 \mu \mathrm{m}$ and $60 \mu \mathrm{m})$. Apart from these parameters, two cooling types were used: conventional cooling and MQL (Minimum Quantity Lubricant). The results obtained through the roughness of the grinded surface and statistical analyzes showed a reduction in the roughness of the parts with small and medium grain size when using MQL cooling. The ceramics with small grain size grinded with a table speed of $55 \mathrm{~mm} / \mathrm{s}$ and depth of cut of $60 \mu \mathrm{m}$ showed roughness of less than $0.28 \mu \mathrm{m}$ using the MQL cooling system. However, when grinded at higher speeds using MQL the ceramics with smaller grain size showed an increase in surface roughness values.
\end{abstract}

Keywords: ceramics, alumina, grinding. 


\section{INTRODUÇÃO}

Materiais cerâmicos apesar da sua elevada fragilidade são candidatos atrativos para algumas aplicações, pois apresentam excelentes propriedades mecânicas e um desempenho excepcional [1,2].

Cerâmicas avançadas estão ligadas à tecnologia de ponta, tendo propriedades elétricas, magnéticas e ópticas muito particulares. Esses materiais são amplamente utilizados em sistemas de comunicação por fibras ópticas, sistemas microeletromecânicos, como rolamentos de esferas, em aplicações que exploram o comportamento piezoelétrico e em aplicações como biomateriais. A ISO (International Organization for Standardization) determina os valores apropriados de rugosidade superficial e outras propriedades físicas que os materiais cerâmicos e, especificamente as cerâmicas comerciais de alumina, devem apresentar para poderem ser aplicados como implantes e funcionarem adequadamente [3].

Suas propriedades mecânicas são um grande fator limitante para sua utilização, tendo como desvantagem principal o fato de estar sujeita a fraturas catastróficas de uma maneira frágil, havendo uma pequena absorção de energia [3]. Essa fragilidade está relacionada com sua baixa capacidade de se deformar plasticamente, isso ocorre, pois a cerâmica apresenta uma estrutura cristalina pouco simétrica mesmo com o aumento de temperatura próximo ao ponto de fusão, não resultando na ativação de mais do que dois ou três sistemas de deslizamento de discordâncias. Assim, há pouca deformação plástica e a elevada dureza persiste mesmo em altas temperaturas, ao contrário dos metais. Quando ocorre a deformação plástica em cerâmicas, normalmente ela é seguida de uma trinca ou mesmo ruptura $[4,5]$.

Porém, o sucesso da cerâmica na maioria das aplicações depende não somente das propriedades do material e do projeto da peça, mas também da qualidade do produto usinado, em termos de acabamento superficial, tensões residuais e defeitos [6].

Sua utilização em diversas áreas é limitada pela grande dificuldade em usinar este material, assim como pelo alto custo do processamento. Consequentemente, é necessário o desenvolvimento de novas técnicas de usinagem que sejam eficientes e economicamente viáveis para o processamento de cerâmicas de engenharia [7].

Em cerâmicas avançadas o processo de fabricação é mais rigoroso e o grau de pureza da sua matéria prima é altamente elevado, uma vez que sua aplicação almeja melhores propriedades mecânicas. Nesta classe têm-se as biocerâmicas, ou seja, cerâmicas que exigem um alto grau de resistência à tração, à compressão e elevada dureza.

O processo de retificação em cerâmicas é uma técnica que apresenta um elevado grau de complexidade, uma vez que esse material apresenta alta dureza e fragilidade após a sinterização. Para poder realizar este processo, normalmente se faz necessário a utilização de um rebolo diamantado, pois esta é a forma mais eficaz na usinagem de precisão de materiais não-metálicos frágeis como as cerâmicas $[1,2,8]$.

A complexidade da retificação é consequência da grande quantidade de arestas de corte dos grãos, da aleatoriedade dos seus formatos e da contínua alteração da geometria das arestas durante a retificação, devido ao desgaste e fratura dos mesmos [9]. O processo é de grande importância na cadeia produtiva, pois interfere diretamente na rugosidade da peça, influenciando assim, o acabamento superficial e afetando as características finais da peça [10].

Parâmetros importantes na definição do processo de retificação são a taxa específica de remoção de material e a espessura equivalente de corte.

A taxa específica de remoção de material $\left(\mathrm{Q}_{\mathrm{w}}{ }_{\mathrm{w}}\right)$ é dada por:

$Q^{\prime}{ }_{w}=a_{p} \times v_{w}$

onde: $Q^{\prime}{ }_{w}=$ taxa específica de remoção de material $\left[\mathrm{mm}^{2} / \mathrm{s}\right]$;

$\mathrm{a}_{\mathrm{p}}=$ profundidade de corte $[\mu \mathrm{m}]$;

$\mathrm{v}_{\mathrm{w}}=$ velocidade da peça ou mesa $[\mathrm{mm} / \mathrm{s}]$.

A espessura equivalente de corte $\left(\mathrm{h}_{\mathrm{eq}}\right)$ representa a espessura da camada de material removida pelo rebolo em uma volta completa e é dada por:

$h_{e q}=\frac{a_{p} \times v_{w}}{v_{s}}$

onde: $\mathrm{h}_{\mathrm{eq}}=$ espessura equivalente de corte $[\mu \mathrm{m}]$;

$\mathrm{v}_{\mathrm{s}}=$ velocidade de corte $[\mathrm{m} / \mathrm{s}]$.

A espessura equivalente de corte calcula a espessura de material que é removido, sendo assim, está totalmente ligada à rugosidade da peça.

$\mathrm{O}$ aumento de $\mathrm{h}_{\mathrm{eq}}$, seja pelo aumento da profundidade de corte ou da velocidade da peça, ou pela redu- 
ção da velocidade de corte, causa aumento dos esforços de corte podendo reduzir o tempo de vida do rebolo e também aumento da rugosidade [14].

A rugosidade é um fator muito importante a ser analisado, pois irregularidades superficiais atuam como concentradores de tensão, influenciando, portanto, as propriedades mecânicas das peças acabadas.

A partir do momento em que o rebolo toca a superfície a ser usinada e durante o movimento do rebolo sobre a peça, pequenos pedaços de material são removidos em forma de cavaco e defeitos são gerados nessa superfície. A formação de cavacos está intimamente ligada às propriedades e estrutura microscópica do material, parâmetros de retificação, características do rebolo e mecanismos de remoção. Fatores que estão diretamente ligados a qualidade final da peça [1].

O mecanismo de remoção de material geralmente é classificado em duas classes: fratura frágil e deformação plástica ou dúctil. A fratura frágil é similar à indentação de materiais frágeis, apresentam trincas laterais que são responsáveis pela remoção de materiais e trincas médias que reduzem a resistência do material. Na fratura frágil ocorrem nucleação e propagação de trincas, cavacos ou riscos $[1,2,8,11]$.

A deformação plástica ou regime dúctil é equivalente à formação de cavaco em metais, envolvendo riscamento e formação de cavaco. O que controla o processo de fratura frágil ou deformação plástica é a resistência mecânica, a dureza, tenacidade à fratura e a microestrutura do material $[1,2,8,12]$.

No caso da alumina o mecanismo de remoção de material é frágil, já no caso da zircônia, outra cerâmica muito utilizada pelas suas excelentes propriedades mecânicas, o regime de remoção de material prevalecente é dúctil, apesar de existir também regime frágil no processo de geração de cavaco [1, 13].

Além dos parâmetros como velocidade e profundidade de corte durante a retificação, outro parâmetro que influência o acabamento superficial da peça é o tipo de sistema de lubri-refrigeração, podendo o fluido ser óleo integral ou uma mistura de óleo e água e o sistema otimizado, convencional dentre outros. É mais recomendável utilizar o fluido miscível em água, podendo obtê-lo como uma emulsão ou como uma solução. O fluido tem a função de refrigerar o sistema (rebolo/peça/máquina) e expulsar o cavaco da região de corte [14]. Desta forma, ajuda no aumento da produtividade e redução da rugosidade superficial, tornando possível a utilização de velocidades de corte mais altas com maiores taxas de remoção e profundidades de corte, entretanto isso só ocorre quando feita de modo adequado [16].

Dentre esses tipos de fluidos existentes, existem três técnicas adotadas: convencional, consiste na aplicação do fluido de corte com alta vazão e baixa velocidade; otimizada, utiliza uma vazão menor do que na convencional, mas a velocidade de aplicação é feita com o mesmo valor da velocidade periférica do rebolo; e MQL, onde existe a aplicação de pouca quantidade de óleo lubrificante atomizado com ar comprimido diretamente na região de usinagem [15]. As duas primeiras técnicas utilizam como fluido uma mistura de óleo e água, enquanto que o MQL utiliza apenas óleo.

Neste trabalho procurou-se estudar o efeito da velocidade da peça, da profundidade de corte e do sistema de lubri-refrigeração durante a retificação no acabamento superficial de cerâmicas de alta alumina fabricadas com três diferentes granulometrias.

\section{MATERIAIS E MÉTODOS}

As barras cerâmicas de alta alumina, utilizadas neste trabalho, foram fornecidas pela CoorsTek, com dimensões $150 \times 25 \times 10 \mathrm{~mm}$, especialmente fabricadas com três tipos de granulometria para este trabalho, sendo grãos grossos $(3,0-3,5 \mu \mathrm{m})$, médios $(2,0-2,5 \mu \mathrm{m})$ e finos $(1,8-2,0 \mu \mathrm{m})$.

Para confecção dos ensaios foi utilizado uma retificadora plana da marca Sulmecânica modelo RAPH - 1055E, onde o rebolo permaneceu com velocidade de corte constante de $33 \mathrm{~m} / \mathrm{s}$ e foram variados os seguintes parâmetros de retificação: velocidade da peça em $55 \mathrm{~mm} / \mathrm{s}$ e $240 \mathrm{~mm} / \mathrm{s}$; profundidade de corte em $5 \mu \mathrm{m}$ e $60 \mu \mathrm{m}$.

Durante a retificação foram adotadas duas condições de lubri-refrigeração, sendo a primeira convencional empregando um fluido de corte da marca CLEAN 2115AL - ATF soluções - Master Chemical Corp, com concentração de 5\% (e 95\% de água) e vazão de 27,5 L/min. Na segunda condição utilizou-se a técnica MQL com fluido da empresa Accu-Lube LB 2000 e vazão de 70 mL/h. 
A dressagem foi realizada após cada tratamento, sendo que foram realizados 10 passes de $20 \mu \mathrm{m}$ de profundidade em cada passe para garantir que o rebolo tivesse as mesmas condições iniciais em todos os tratamentos. A Figura 1 esquematiza o processo de confecção experimental adotado.
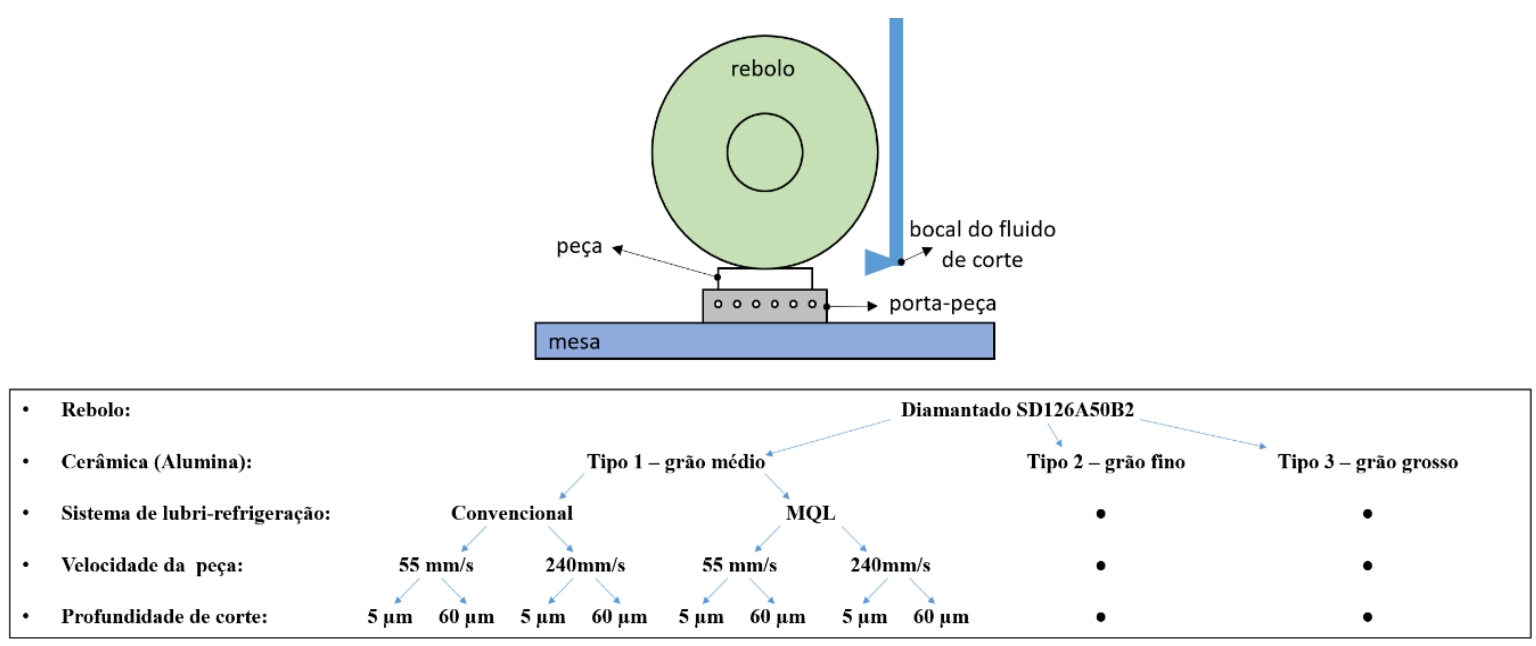

Figura 1: Fluxograma de ensaios.

A taxa de remoção específica de corte e espessura equivalente de corte foram calculadas através das equações (1) e (2) e estão listadas nas Tabelas 1.

Tabela 1: Valores calculados da taxa especifica de remoção de material e espessura equivalente de corte a partir da velocidade da peça e profundidade de corte.

\begin{tabular}{llll}
$\mathbf{a}_{\mathbf{p}}[\boldsymbol{\mu m}]$ & $\mathbf{V}_{\mathbf{w}}[\mathbf{m m} / \mathbf{s}]$ & $\mathbf{h}_{\mathrm{eq}}[\boldsymbol{\mu m}]$ & $\mathbf{Q}_{\mathbf{w}}{ }^{\prime}\left[\mathbf{m m}^{2} / \mathbf{s}\right]$ \\
\hline $\mathbf{5}$ & 55 & 0,008 & 0,275 \\
\hline $\mathbf{5}$ & 240 & 0,035 & 1,2 \\
\hline $\mathbf{6 0}$ & 55 & 0,097 & 3,3 \\
\hline $\mathbf{6 0}$ & 240 & 0,423 & 14,4 \\
\hline
\end{tabular}

A partir dos valores obtidos, pode-se observar que quanto maior a velocidade e a profundidade de corte, maior a espessura equivalente de corte e maior a taxa de remoção. Quanto maior a taxa de remoção menor o tempo de ciclo, ou seja, menor o tempo para retificar as peças cerâmicas, com o aumento da taxa de remoção e da velocidade, aumenta o volume de material removido da superfície da peça retificada, podendo causar mais danos à peça. Logo, se houver aumento da espessura de corte, o acabamento superficial da peça será prejudicado.

A rugosidade foi medida em um rugosímetro portátil Taylor-Hobson modelo Surtronic 3+, onde seu cut-off ficou fixado em $0,25 \mathrm{~mm}$ e calibrado no parâmetro Ra. Para obter precisão nos resultados de rugosidade, dividiu-se a superfície retificada em 6 pontos (foram feitas 3 medidas para cada ponto escolhido) mapeando toda região da peça.

Um estudo estatístico foi aplicado para evidenciar qual parâmetro teve maior influência sobre o acabamento superficial. Utilizou-se uma análise fatorial através do Software Minitab, baseada na análise de variância ANOVA e diagramas de Pareto, determinando assim a influência de cada fator na qualidade superficial.

\section{RESULTADOS E DISCUSSÕES}

A Tabela 2 traz as médias aritméticas das medidas de rugosidade realizadas na peça cerâmica após os testes de retificação. 
Tabela 2: Rugosidade média das peças.

\begin{tabular}{|c|c|c|c|c|}
\hline Tipo Material & Lubri-refrigeração & $\mathbf{v}_{\mathbf{w}}[\mathbf{m m} / \mathbf{s}]$ & $a_{p}[\mu m]$ & $\mathbf{R}_{\mathrm{a}}$ média $[\mu \mathrm{m}]$ \\
\hline \multirow{8}{*}{ Tipo 1} & \multirow{4}{*}{ Convencional } & \multirow{2}{*}{55} & 5 & 0,54 \\
\hline & & & 60 & 0,64 \\
\hline & & \multirow{2}{*}{240} & 5 & 0,56 \\
\hline & & & 60 & 0,45 \\
\hline & \multirow{4}{*}{ MQL } & \multirow{2}{*}{55} & 5 & 0,41 \\
\hline & & & 60 & 0,44 \\
\hline & & \multirow{2}{*}{240} & 5 & 0,49 \\
\hline & & & 60 & 0,52 \\
\hline \multirow{8}{*}{ Tipo 2} & \multirow{4}{*}{ Convencional } & \multirow{2}{*}{55} & 5 & 0,45 \\
\hline & & & 60 & 0,47 \\
\hline & & \multirow{2}{*}{240} & 5 & 0,42 \\
\hline & & & 60 & 0,46 \\
\hline & \multirow{4}{*}{ MQL } & \multirow{2}{*}{55} & 5 & 0,35 \\
\hline & & & 60 & 0,28 \\
\hline & & \multirow{2}{*}{240} & 5 & 0,43 \\
\hline & & & 60 & 0,54 \\
\hline \multirow{8}{*}{ Tipo 3} & \multirow{4}{*}{ Convencional } & \multirow{2}{*}{55} & 5 & 0,68 \\
\hline & & & 60 & 0,73 \\
\hline & & \multirow{2}{*}{240} & 5 & 0,65 \\
\hline & & & 60 & 0,67 \\
\hline & \multirow{4}{*}{ MQL } & \multirow{2}{*}{55} & 5 & 0,61 \\
\hline & & & 60 & 0,67 \\
\hline & & \multirow{2}{*}{240} & 5 & 0,66 \\
\hline & & & 60 & 0,77 \\
\hline
\end{tabular}

Através da tabela é possível observar que a rugosidade não ultrapassou $0,8 \mu \mathrm{m}$ mesmo quando se elevou a velocidade. A Figura 2 traz os gráficos dos resultados de rugosidade versus profundidade de corte para ambas as velocidades.

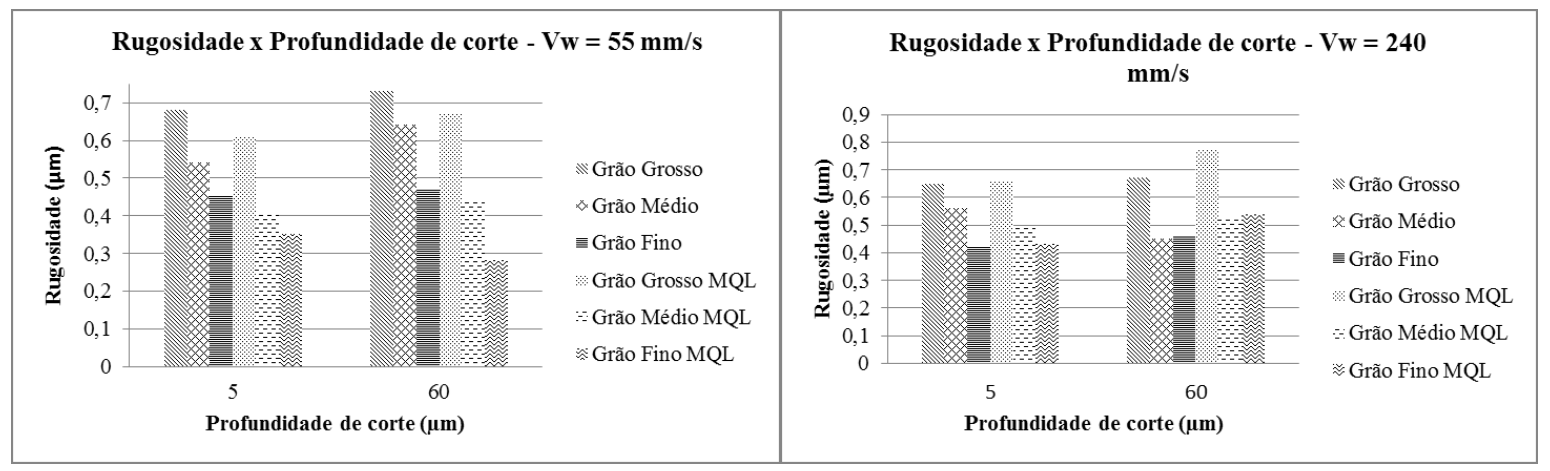

Figura 2: Média da rugosidade superficial da superfície retificada da cerâmica em função da profundidade de corte.

Observando-se a Figura 2, referente a velocidade da peça de $55 \mathrm{~mm} / \mathrm{s}$, é possível afirmar que houve uma tendência de diminuição da rugosidade nos resultados obtidos para a condição mais branda, ou seja, a rugosidade se comportou como esperado para as três granulometrias em ambos os fluidos. Houve um decréscimo de acordo com o grão, sendo que o grão grosso apresentou maior rugosidade o grão médio uma rugosidade intermediária e o grão fino uma rugosidade baixa, sendo ela de $0,28 \mu \mathrm{m}$ quando empregado MQL como lubrificante, mesmo quando a profundidade de corte foi de $60 \mu \mathrm{m}$.

Com o aumento da velocidade da peça $(240 \mathrm{~mm} / \mathrm{s})$ e da profundidade de corte, o emprego do sistema de lubri-refrigeração por MQL não se mostrou tão eficiente mesmo para as cerâmicas de menor granulometria. A rugosidade medida nas cerâmicas retificadas com profundidade de corte de $60 \mu \mathrm{m}$ e $55 \mathrm{~mm} / \mathrm{s}$ foi de $0,28 \mu \mathrm{m}$, enquanto que naquelas retificadas com velocidade de $240 \mathrm{~mm} / \mathrm{s}$ foi de $0,54 \mu \mathrm{m}$, quando o sistema MQL foi empregado.

Quando se compara a eficiência dos fluidos, observa-se um aproveitamento melhor para o MQL para 
velocidade de $55 \mathrm{~mm} / \mathrm{s}$. A Figura 3 resume os resultados de rugosidade, exibindo-os em função da espessura equivalente de corte.

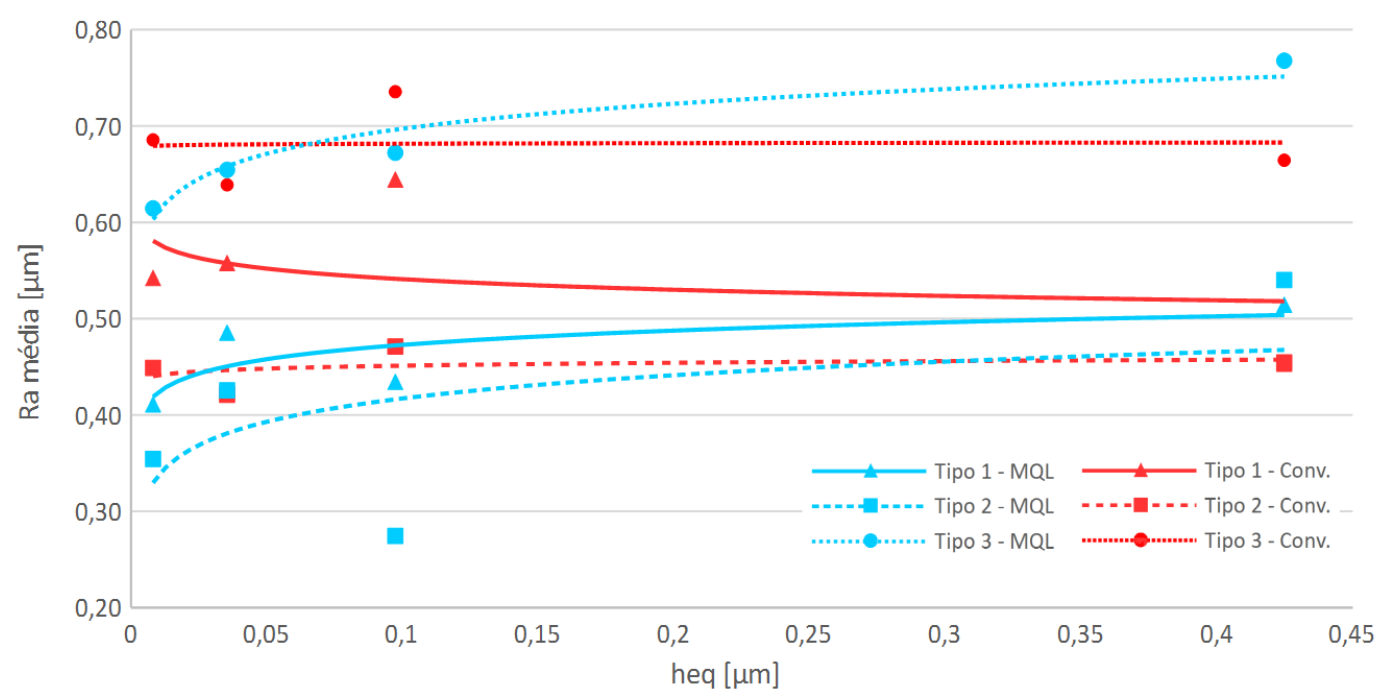

Figura 3: Média da rugosidade superficial da superfície retificada da cerâmica em função da espessura equivalente de corte.

Pode-se perceber que o efeito do aumento da espessura equivalente de corte provoca uma piora nos valores de rugosidade nos ensaios que se utilizaram da técnica de MQL, indicando que o cavaco pode estar se alojando nos poros do rebolo. Este efeito causa o aumento da rugosidade, pois o rebolo permanece "sujo" durante o corte subsequente. $\mathrm{O}$ efeito não é observado na condição de lubri-refrigeração convencional.

Buscando interpretar melhor os resultados sobre quais parâmetros influenciaram o acabamento final das peças, empregou-se métodos estatísticos que auxiliaram na construção do diagrama de Pareto.

Este estudo inicialmente avaliou individualmente os parâmetros e depois fez combinações para ver qual combinação apresentou maior influência na rugosidade. A Figura 4 exibe a influência de cada um dos parâmetros na rugosidade de maneira individual.

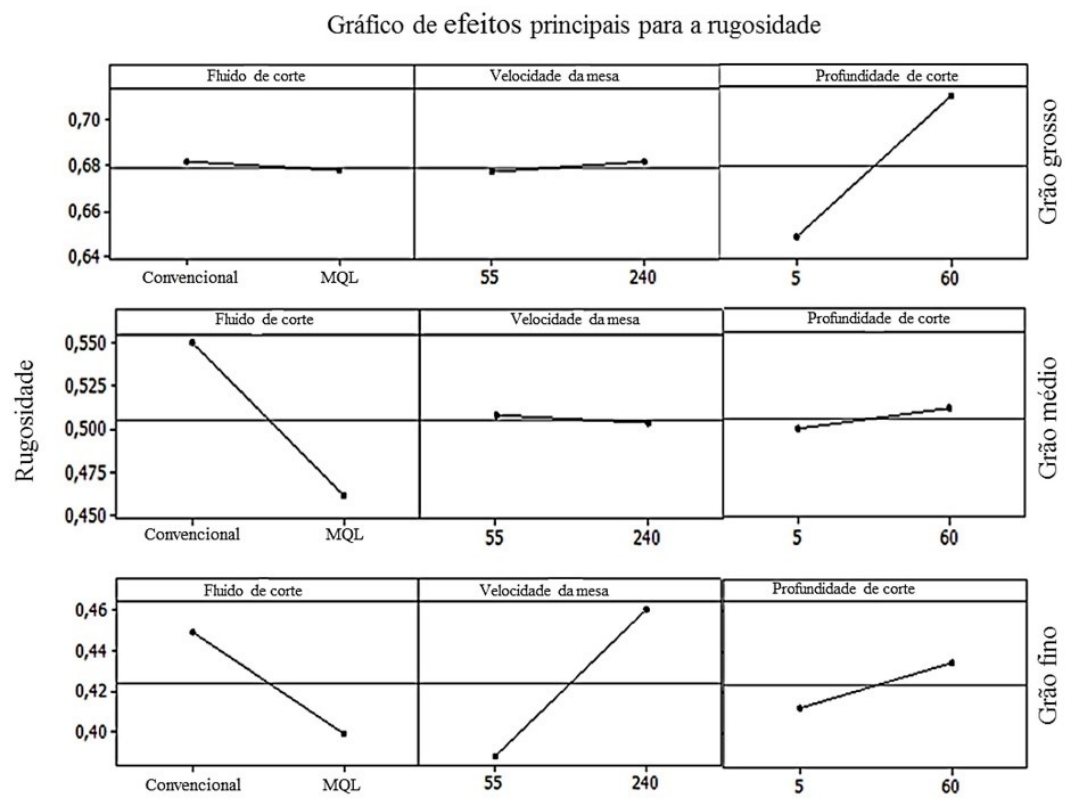

Figura 4: Gráfico de efeitos principais para a rugosidade para cerâmicas de grão grosso, médio e fino.

É possível observar que para a cerâmica de grão grosso o parâmetro que mais influenciou foi à profundidade de corte de $60 \mu \mathrm{m}$. Comparando com os resultados da Tabela 2e Figura4, pode-se notar que a 
rugosidade para essa peça manteve-se alta, e também que esta apresentou o pico mais alto de rugosidade sendo esse de $0,73 \mu \mathrm{m}$, comportamento diferente para as outras cerâmicas.

Para a cerâmica de grão médio o fator que mais influenciou foi o fluido de corte, sendo o MQL o que apresentou menor rugosidade, associando os resultados é possível observar que o MQL para essa cerâmica só não se apresentou satisfatório para a profundidade de $60 \mu \mathrm{m}$ na velocidade de $240 \mathrm{~mm} / \mathrm{s}$. Por esta cerâmica ser de grão médio quando comparada com as outras ela sempre se manteve no intermédio dos resultados.

Já para a cerâmica de grão fino todos os fatores influenciaram em sua rugosidade principalmente a velocidade da peça e o fluido de corte, o que também é observado na Figura 2 e na Tabela 2, esta cerâmica teve a menor rugosidade durante a condição mais branda, e teve rugosidade superior a cerâmica de grão médio na condição mais agressiva quando o fluido empregado foi o MQL.

O Diagrama de Pareto (Figura 5) confirma a profundidade como principal efeito no acabamento. Além disso, mostra que a interação entre fluido e velocidade da peça também exercem impacto significativo sobre a rugosidade. Neste gráfico todos os fatores e suas combinações com efeitos padronizados maiores que 1,978 são significativos, ou seja, a sua mudança de magnitude gera variação suficiente na variável de saída (rugosidade).

Através do diagrama é possível afirmar que para a cerâmica de grão grosso a combinação entre fluido de corte (seja ele convencional ou MQL) com a velocidade da peça e a profundidade de corte, foram os fatores que exerceram o impacto mais significativo sobre a rugosidade, o que já foi observado em resultados anteriores.

Para a cerâmica de grão médio o diagrama mostra que os efeitos que menos influenciaram a rugosidade foram: a velocidade da peça, a profundidade de corte e a combinação, fluido e profundidade de corte. Como visto nos resultados anteriores, a cerâmica com granulometria média não mostrou alterações significativas quando comparadas com as outras cerâmicas.

No caso das cerâmicas com granulometria mais fina houve uma associação de parâmetros que influenciaram significativamente a rugosidade superficial das peças retificadas, tais como: a combinação entre sistema de lubri-refrigeração e a velocidade da peça, ou mesa (FV); velocidade da peça e profundidade de corte (VA) e uma combinação dos três fatores (FVA). Neste caso, o único fator que não influenciou significativamente a rugosidade da superfície retificada foi à combinação entre sistema de lubri-refrigeração e profundidade de corte (FA).
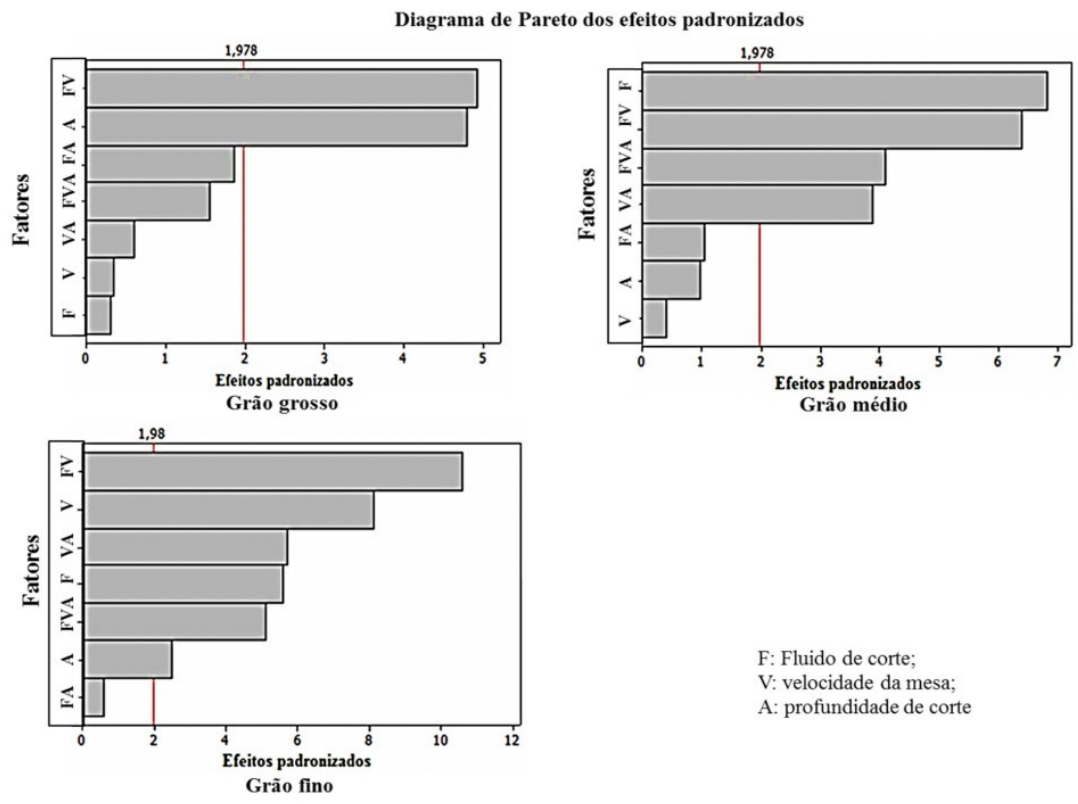

Figura 5: Diagrama de Pareto para as cerâmicas de grão grosso, médio e fino.

Portanto, dos resultados obtidos na Figura 2 e na Tabela 2, nota-se que para o grão grosso, o fluido MQL apresenta resultados mais satisfatórios quando a velocidade é mais baixa, porém, com o aumento da velocidade, há um aumento da rugosidade superficial. Entretanto, quando existe a aplicação do fluido convencional ocorre o fenômeno oposto, ou seja, ocorreu uma redução na rugosidade com aumento da velocidade da peça.

Com o aumento da profundidade de corte, ocorre uma piora na rugosidade superficial para ambos os fluidos de corte, havendo uma tendência para o aumento de rugosidade para os testes de MQL.

Para ambas as velocidades da peça há um aumento de rugosidade com o aumento da profundidade de corte. 
Prevalecendo também a remoção frágil de material, provocada pela presença de microtrincas.

Assim como ocorre no grão grosso, ocorre no grão médio, o aumento da velocidade da peça faz a rugosidade aumentar quando existe a aplicação do MQL. Isso se deve à baixa capacidade de refrigeração do MQL, gerando uma tendência de aumento de temperatura e piora no acabamento com o aumento na rugosidade superficial. Com o aumento da profundidade de corte não há alteração superficial significativa para nenhum dos fluidos, sendo também o melhor acabamento apresentado pelo MQL [14].

Além desses fatores enunciados, durante a lubri-refrigeração empregando a técnica do MQL, notou-se a formação de uma "borra" de difícil remoção resultante da mistura do fluido de corte, cavaco e grãos do rebolo. Esta borra de cavaco remanescente na zona de corte pode afetar consideravelmente os valores de rugosidade da peça [4].

Para baixas profundidades de corte, a velocidade de $55 \mathrm{~mm} / \mathrm{s}$ é mais vantajosa para o processo em termos de rugosidade, porém essa situação se inverte para profundidades mais altas, onde se torna mais vantajosa a velocidade de $240 \mathrm{~mm} / \mathrm{s}$. Isso pode ter ocorrido pelo fato de velocidades altas com profundidades baixas gerarem menores superfícies de contato entre o rebolo e peça, uma maior elevação da temperatura e consequentemente, um pior acabamento superficial.

Já para o grão fino com o aumento da velocidade da peça, não há grande variação da rugosidade para o fluido convencional. Fato este, que não se repete para o fluido MQL, onde há um aumento expressivo da rugosidade superficial com o aumento da velocidade. Ocorrendo da mesma forma que na peça de grão médio, ou seja, mais uma vez a baixa refrigeração do MQL ocasionou aumento de rugosidade. Ao se analisar o aumento da velocidade da peça em relação à profundidade de corte, observa-se que não há mudança expressiva do acabamento superficial para nenhum dos dois tipos de fluido.

Para baixas profundidades de corte não há grande diferença entre utilização de uma velocidade da peça mais baixa ou mais alta. Porém, com o aumento da profundidade de corte, a velocidade de $240 \mathrm{~mm} / \mathrm{s}$ tende a gerar um aumento da rugosidade, enquanto a velocidade de $55 \mathrm{~mm} / \mathrm{s}$ tende a reduzi-la. Para este tipo de peça, remoções de material mais agressivas ocasionaram um expressivo aumento de rugosidade, provavelmente pela remoção de material em regime frágil.

Apesar dos processos de deformação plástica e remoção de material por fratura frágil poderem ocorrer durante o processo de retificação de cerâmicas, o processo de propagação de trincas ao longo dos contornos de grãos (microfratura) e remoção de material em escala de grão são mais comuns, justificando os resultados obtidos nas diferentes peças analisadas, pois quanto maiores os grãos, maiores os picos e vales da superfície após a usinagem.

\section{CONCLUSÕES}

De acordo com o presente estudo, é possível concluir que a retificação é um processo adequado quando utilizado em cerâmicas, de modo geral, os resultados permaneceram satisfatórios e a rugosidade das peças permaneceu inferior a $0,8 \mu \mathrm{m}$.

Fazendo uma abordagem geral, para os três tipos de peça analisados, o fluido MQL apresenta inicialmente resultados satisfatórios, porém com o aumento da velocidade da peça de $55 \mathrm{~mm} / \mathrm{s}$ para $240 \mathrm{~mm} / \mathrm{s}$ os valores de rugosidade tendem a aumentar. Isso possivelmente ocorre devido a baixa capacidade de refrigeração deste tipo de fluido, ou seja, com o aumento da velocidade da peça existe um aumento do atrito entre a cerâmica e o rebolo e por consequência desse atrito, há o aumento da temperatura do sistema. Este tipo de fluido apresenta uma excelente capacidade de lubrificação, mas baixa capacidade de refrigeração, pois o ar faz parte de sua composição, diferente do fluido convencional, que leva água na mistura. Sendo assim, o fluido MQL apresenta baixa condutividade térmica, tornando-se inviável para altas velocidades de retificação.

Além disso, os estudos estatísticos realizados nesta etapa do trabalho concluíram que houve uma redução na rugosidade das peças com granulometria media e fina quando se utilizou a lubri-refrigeração MQL. As cerâmicas de granulometria fina, retificadas com velocidade de $55 \mathrm{~mm} / \mathrm{s}$ e profundidade de $60 \mu \mathrm{m}$, apresentaram rugosidade inferior a $0,28 \mu \mathrm{m}$ usando o sistema de lubri-refrigeração MQL. Porém quando retificadas em velocidades maiores utilizando MQL as cerâmicas de granulometria mais fina apresentaram um aumento nos valores de rugosidade.

\section{BIBLIOGRAFIA}

[1] ZHANG, D., LI, C., JIA, D., ZHANG, Y., "Investigation into Engineering Ceramics Grinding Mechanism and the Influential Factors of the Grinding Force", International Journal of Control and Automation, v.7, n.4, pp.19-34, 2014. 
[2] ZHENG, X., ZHAO, F., ZHANG, J., "Mechanical Properties and Fracture Behaviour of Multilayer Alumina Composites", Journal of Wuhan University of Technology-Mater. Sci., pp. 965- 967, Oct. 2015.

[3] HENCH, L. L. "An Introduction to Bioceramics", 2nd Edition, Imperial College Press, 2013, cap. 2., In: HULBERT, S. F. The use of Alumina and Zirconia in Surgical Implants.

[4] OIKAWA, M. H., BIANCHI, E. C., DESTRO, R. S., et al., "Cerâmicas avançadas no processo de retificação cilíndrica externa de mergulho com rebolos diamantados com a técnica da mímima quantidade de lubrificação e refrigeração otimizada", Cerâmica, v.57, pp.263-273, 2011.

[5] ORÉFICE, R.L., PEREIRA, M.M., MANSUR, H.S., Biomateriais, Fundamentos \& aplicações., UFMG, MG, Editora Cultura Médica, 2008.

[6] FUJITA, H. Retificação da alumina com rebolo diamantado usando diferentes métodos de lubrirefrigeração. Dissertação de M.Sc., Faculdade de Engenharia de Bauru, Universidade Estadual Paulista, Unesp, Bauru, Brasil, 2007.

[7] EMAMI, M., SADEGHI, M.H., SARHAN, A.A.D. "Investigating the effects of liquid atomization and delivery parameters of minimum quantity lubrication on the grinding process of $\mathrm{Al}_{2} \mathrm{O}_{3}$ engineering ceramics", Journal of Manufacturing Processes, v.15, n. 3, pp. 374-388, 2013.

[8] AGARWAL, S., RAO, P. V., "Experimental investigation of surface/subsurface damage formation and material removal mechanisms in $\mathrm{SiC}$ grinding", International Journal of Machine Tools \& Manufacture, v.48, pp. 698-710, 2008.

[9] TÖNSHOFF, H.K., LIERSE, T., INASAKI, I., "Grinding of advanced ceramics", In: JAHANMIR, S., RAMULU, M., KOSHY, P., "Machining of Ceramics and Composites", cap.3, 1999.

[10] DHANASEKAR, B., MOHAN, N. K., BHADURI, B., et al. "Evaluation of surface roughness based on monochromatic speckle correlation using image processing", Precision Engineering, v. 32, n. 3, p. $196-$ 206, 2008.

[11] XU, H. K.., JAHANMIR, S. "Microfracture and material removal in scratching of alumina", Journal of Materials Science, v.30, pp. 2235-2247, 1995.

[12] XU, H.H.K., JAHANMIR, S., WANG, Y., "Effect of grain size on scratch interactions and material removal in alumina", Journal of American ceramic society, v.78, n.9, p.2443-2448, 1995.

[13] HUANG, H.; LIU, Y.C., "Experimental investigations of machining characteristics and removal mechanisms of advanced ceramics in high speed deep grinding", International Journal of Machine Tools \& Manufacture, v. 43, p.811-23, 2003.

[14] DINIZ, A. E., MARCONDES, F. C., COPPINI, N.L, “Tecnologia da usinagem dos materiais”, 7 ed., São Paulo, Brasil, Artliber Editora LTDA, 2010.

[15] SOUSA, R. M., ICERI D. M., DESTRO, R. S., et al., "Retificação de cerâmica de alumina relacionada com diferentes técnicas de lubri-refrigeração: convencional e mínima quantidade de lubrificação", Cerâmica, v.57, pp. 76-84, 2011.

[16] BIANCHI, E. C., DINIZ, A. E., PEREIRA, M. G., et al., "Limpeza da superfície de corte de rebolos por um sistema de ar comprimido na retificação cilíndrica externa de mergulho de materiais cerâmicos refrigerados com a técnica da mínima quantidade de lubrificante (MQL)", Cerâmica, v. 58, pp. 270-274, 2012. 